\title{
In vitro and in vivo rationale for the triple combination of panobinostat (LBH589) and dexamethasone with either bortezomib or lenalidomide in multiple myeloma
}

\author{
Enrique M. Ocio, $, 1,2$ David Vilanova, ${ }^{1}$ Peter Atadja, ${ }^{3}$ Patricia Maiso, ${ }^{1}$ Edvan Crusoe,,${ }^{1,4}$ Diego Fernández-Lázaro, ${ }^{1}$ \\ Mercedes Garayoa, ${ }^{1}$ Laura San-Segundo, ${ }^{1}$ Teresa Hernández-Iglesias, ${ }^{1}$ Enrique de Álava, ${ }^{1}$ Wenlin Shao, ${ }^{3}$ \\ Yung-Mae Yao, ${ }^{3}$ Atanasio Pandiella, ${ }^{1}$ and Jesús F. San-Miguel ${ }^{1,2}$
}

${ }^{1}$ Centro de Investigación del Cáncer, IBMCC/CSIC-Universidad de Salamanca, Spain; ${ }^{2}$ Department of Hematology, University Hospital of Salamanca, Salamanca, Spain, and ${ }^{3}$ Novartis Institutes for Biomedical Research, Cambridge, MA, USA

\section{ABSTRACT}

\section{Background}

Combinations of drug treatments based on bortezomib or lenalidomide plus steroids have resulted in very high response rates in multiple myeloma. However, most patients still relapse, indicating the need for novel combination partners to increase duration of response or to treat relapsed disease. We explored the antimyeloma activity of triple combinations of these wellestablished schemes with panobinostat, a novel deacetylase inhibitor with a multi-targeted profile.

\section{Design and Methods}

The activity of these combinations was explored in vitro in cell lines by using MTT and annexin $\mathrm{V}$, ex vivo by flow cytometry, and in vivo using two different murine models of human myeloma: one bearing a subcutaneous plasmacytoma and another with a disseminated myeloma. Moreover, gene expression profiling and immunohistochemical studies were performed.

\section{Results}

The addition of panobinostat (LBH589) to dexamethasone and either bortezomib or lenalidomide resulted in clear potentiation in multiple myeloma cell lines, freshly isolated plasma cells, and murine models of multiple myeloma. The quantification of the potency of these combinations by using the Chou-Talalay method showed synergistic combination indices for all of them. This effect derived from the deregulation of a cluster of genes that was completely different from the sum of genes affected by the single agents (895 and 1323 genes exclusively deregulated by panobinostat and dexamethasone plus bortezomib or lenalidomide, respectively). Functional experiments, such as annexin V staining, cell cycle analysis, and immunohistochemical studies also supported this potentiation. Anti-myeloma efficacy was confirmed in an extramedullary plasmacytoma model and a disseminated luciferized model, in which panobinostat also provided a marked benefit in bone disease.

\section{Conclusions}

The potent activity, together with the exclusive mechanistic profile, provides the rationale for the clinical evaluation of these drug combinations in multiple myeloma.

Key words: panobinostat, LBH589, multiple myeloma, HDAC inhibitors.

Citation: Ocio EM, Vilanova D, Atadja P, Maiso P, Crusoe E, Fernández-Lázaro D, Garayoa M, San Segundo L, Hernández Iglesias T, de Álava E, Shao W, Yao YM, Pandiella A, and San Miguel $J F$. In vitro and in vivo rationale for the triple combination of panobinostat (LBH589) and dexamethasone with either bortezomib or lenalidomide in multiple myeloma. Haematologica 2010;95:794803; doi:10.3324/haematol.2009.015495

(C)2010 Ferrata Storti Foundation. This is an open-access paper.

\section{$\longrightarrow$ \\ EMO and DV contributed equally to this manuscript \\ Acknowledgments: the authors would like to acknowledge Dina Marenstein of Chameleon Communications International, who provided editorial support with funding from Novartis Oncology.}

Funding: this work was supported by grants from the Ministry of Education and Science of Spain (BFU2006-01813/BMC, RD06/0020/0041, and RD06/0020/0059).

The CIC receives support from the European Community through the regional development funding program (FEDER). This work was also supported by the 'Acción Transversal del Cáncer' project, through an agreement between Instituto de Salud Carlos III (ISCIII), Spanish Ministry of Science and Innovation, and the Cancer Research Foundation of Salamanca University. Our group also receives support from the Junta de Castilla y Léon through 'Ayudas destinadas a financiar programas de actividad investigadora a realizar por grupos de investigación de excelencia de Castilla y León.

Manuscript received on August 7, 2009; revised version arrived on September 22, 2009; manuscript accepted on October 13, 2009.

\section{Correspondence:}

Enrique M. Ocio, Department of Hematology, University Hospital \& Cancer Research Center. University of Salamanca $P^{0}$ San Vicente, 58-182, 37007

Salamanca, Spain.

E-mail: emocio@usal.es

The online version of this article has a Supplementary Appendix. 


\section{Introduction}

In the last decades, one of the most important advances achieved in oncology treatment has been the use of combinations of agents with different mechanisms of action, in order to increase the efficacy of single agents used as monotherapy. As a result, poly-chemotherapy has become the standard of care in most hematologic malignancies. This has been the case for regimens such as CHOP for the treatment of non-Hodgkin's lymphoma, ABVD for Hodgkin's disease, FLAG-Ida for acute myeloid leukemia or VAD for multiple myeloma (MM).

In recent years there has been a rapid development of novel agents targeting tumor-specific mechanisms in MM; however, these targeted compounds have generally shown limited efficacy when used as monotherapy, ${ }^{1}$ probably due to the multi-step process of tumorigenesis. ${ }^{2}$ Preliminary data indicate that the combination of compounds which simultaneously target multiple tumorigenic pathways induces a more effective and lasting anti-tumor response in different malignancies. ${ }^{1,3-6}$ Therefore, there is a need for the development of optimal combinations of these novel agents in order to improve the survival of patients with myeloma.

A relevant oncogenic mechanism which has recently gained attention is the epigenetic modulation of gene expression. Several studies have demonstrated that enzymes involved in epigenetic mechanisms, such as histone deacetylases (HDAC), are deregulated in many cancers, ${ }^{7,8}$ and thus affect the expression of tumor suppressors and oncogenes in cancer cells. The targeting of HDAC activity with inhibitors has demonstrated significant activity in preclinical experiments and has emerged as a promising strategy in the clinical setting, both in terms of efficacy and breadth of activity. ${ }^{9,10}$ Panobinostat (LBH589) is a novel HDAC inhibitor (HDACi) derived from cynnamic acid hydroxamates and has already shown promising clinical anti-tumor activity when administered as a single agent in patients with refractory hematologic malignancies, particularly cutaneous T-cell lymphoma. ${ }^{11,12}$ In MM, we and others have recently reported the potent in vitro antimyeloma activity of this compound in MM cell lines and in primary samples from MM patients; ${ }^{1,14}$ by contrast the clinical activity of HDACi as single agents appears to be rather modest in this disease. ${ }^{15-17}$ Accordingly, the design of clinical trials based on HDACi combinations would be of clear interest in the clinic. These combinations should be based on scientifically solid preclinical data, in order to avoid repetition of past mistakes in drug combinations.

In this report, we have stepped forward in the investigation of the antimyeloma activity of panobinostat by exploring whether triple combinations of some of these antimyeloma agents are more efficacious than double combinations and we have confirmed this antimyeloma efficacy in two different in vivo murine models, including a model for the investigation of the potential effect of panobinostat on bone disease.

\section{Design and Methods}

\section{Reagents and immunochemicals}

Cell culture media, serum, and penicillin-streptomycin were purchased from Invitrogen Corporation (Gaithersburg, MD, USA). Panobinostat (LBH589) was provided by Novartis
Pharmaceuticals (East Hannover, NJ, USA), bortezomib by Millennium Pharmaceuticals, Ltd. (Cambridge, MA, USA; and London, UK), lenalidomide by Celgene (Summit, NJ, USA); dexamethasone and 3-(4,5-dimethylthiazol-2-yl)-2,5-diphenyltetrazolium bromide (MTT) were purchased from Sigma-Aldrich (Madrid, Spain), and the Annexin V-FITC Kit was from Immunostep (Salamanca, Spain). Other generic chemicals were purchased from Sigma Chemical Co., Roche Biochemicals (Mannheim, Germany) or Merck \& Co., Inc. (Darmstadt, Germany).

\section{Cell culture, cell proliferation, cell cycle, and apoptosis analyses}

The MM1S cell line was kindly provided by Steven Rosen (Northwestern University, Chicago, IL, USA) and cultured in RPMI-1640 with L-glutamine and supplemented with antibiotics (penicillin at 100 units $/ \mathrm{mL}$, streptomycin at $100 \mu \mathrm{g} / \mathrm{mL}$ ) and $10 \%$ fetal bovine serum (FBS) at $37^{\circ} \mathrm{C}$ in a humidified atmosphere in the presence of $5 \% \mathrm{CO}_{2} / 95 \%$ air. Bone marrow samples from patients with $\mathrm{MM}$ were aspirated after obtaining informed consent, lysed with ammonium chloride to remove red blood cells, and cultured in the previous conditions with the only exception of the addition of $20 \%$ FBS. The proliferation of MM cell lines was examined using MTT colorimetric assays as described elsewhere. ${ }^{18}$ For cell cycle and apoptosis analyses in patients' samples, bone marrow cells were incubated with the drugs for $18 \mathrm{~h}$. Afterwards, a multiparametric technique, based on the combination of annexin V-FITC and three-color monoclonal antibodies against myeloma-associated antigens (CD38, CD56, and CD45 all from BD Biosciences, San Jose, CA, USA) was performed, allowing the analysis of apoptosis in tumor plasma cells. The detailed methodology has been previously described. ${ }^{14}$

\section{Quantification of the synergism of panobinostat with other antimyeloma agents}

MM1S cells were treated for $72 \mathrm{~h}$ with different doses of panobinostat, bortezomib, lenalidomide and dexamethasone in monotherapy and in double and triple combinations. For this purpose, three different dose combinations were explored for each triple combination keeping a constant ratio between them. The following doses of panobinostat, bortezomib, lenalidomide and dexamethasone (all of them in $\mathrm{nM}$ ) were used: 0.5:1:250:2.5; 1:2:500:5; and 2:4:1000:10, respectively. The potency of the combination was quantitated with the Calcusyn software (Biosoft, Ferguson, MO, USA), which is based on the Chou Talalay method, ${ }^{19}$ that calculates a combination index (CI) with the following interpretation:

$C I>1$ : antagonistic effect, $C I=1$ : additive effect and $C I<1$ synergistic effect

\section{Microarray RNA analyses}

Gene expression profile studies were performed as previously described. ${ }^{14}$ In brief, MM1S cells were treated with different doses of each drug in monotherapy for $48 \mathrm{~h}$ in order to choose the dose of each of them that induced a beginning of apoptosis $(20-25 \%$ cell death, as assessed by annexin V-FITC staining). The selected doses were panobinostat $(7 \mathrm{nM})$, dexamethasone $(0.9 \mu \mathrm{M})$, bortezomib (3 nM), and lenalidomide $(10 \mu \mathrm{M})$. These doses were fixed and used for the two triple combinations (panobinostat + bortezomib + dexamethasone and panobinostat + lenalidomide + dexamethasone), and in this case, the experiment was stopped, again, when $20-25 \%$ of annexin V staining was observed; that was at 24 and $26 \mathrm{~h}$ for each combination, respectively. Duplicates were obtained from each experiment. Cells were harvested, and the total RNA was extracted using TRIzol Reagent (Life Technologies, 
MD, USA) and purified with RNAeasy Mini Kit (Oiagen, CA, USA). RNA integrity was verified with the Agilent 2100 Bioanalyzer (Agilent, CA, USA). Double-stranded cDNA and biotinylated cRNA were synthesized with T7-polyT primer and the BioArray RNA labeling kit (Enzo, NY, USA), respectively. The labeled RNA was then fragmented and hybridized to HG-U133 plus 2.0 oligonucleotide arrays (Affymetrix, CA, USA), which were scanned in a Gene Array Scanner and analyzed using the DNA-Chip Analyzer software (DChip). Greater than 2-fold changes in gene expression were considered significant. Firstly, changes induced by the drugs in monotherapy and in triple combinations as compared with the untreated samples were analyzed, and, in a second step, the changes induced by the triple combinations and not with the drugs in monotherapy were studied.

\section{Animal models}

For the human subcutaneous plasmacytoma model, CB17SCID mice (The Jackson Laboratory, Bar Harbor, ME, USA) were subcutaneously inoculated into the right flank with $3 \times 10^{6} \mathrm{MM} 1 \mathrm{~S}$ cells in $100 \mu \mathrm{L}$ of RPMI-1640 medium and $100 \mu \mathrm{L}$ of Matrigel (BD Biosciences, San Jose, CA USA). When tumors became palpable, mice were randomized to the control group (receiving the vehicle alone-PBS) or the different treatment groups. At least eight mice were included in each group of randomization. Caliper measurements of the tumor diameters were performed 3 days weekly, and the tumor volume was estimated as the volume of an ellipse using the following formula:

$$
V=4 / 3 \pi \times(a / 2) \times(b / 2)^{2}
$$

where $a$ and $b$ correspond to the longest and shortest diameter respectively. Differences in tumor volumes between groups were evaluated using one-way analysis of variance by using a genera linear model of repeated measures. For survival evaluation, mice were sacrificed when their tumor diameters reached $2 \mathrm{~cm}$, or when they became moribund. Time to clinical endpoint (TTE) was estimated from the day of initiation of treatment, and statistical differences were assessed by Kaplan-Meier curves with the log rank test. Statistical analyses were performed with the SPSS15.0 software (SPSS Inc. Chicago, IL, USA), and statistical significance was defined as $P<0.05$.

A systemic $\mathrm{MM}$ xenograft mouse model was generated by i.v. injection of $2 \times 10^{6} \mathrm{MM} 1 \mathrm{~S}$ cells stably expressing the luciferase protein through the tail vein. Tumor burden was determined from whole animal bioluminescence measured using a Xenogen IVIS200 and Living Image software. Animals were monitored daily for onset of MM clinical signs. The clinical endpoint was defined as changes in posture, gait, or mobility that interfere with feeding behavior, such as hind limb paralysis, consistent foot dragging, or spinal curvature. Compound activity was determined by tumor growth inhibition (TGI) and TTE. TGI was defined as the percent change in tumor burden of treated over control animals $(\% \Delta \mathrm{T} / \mathrm{C})$ on day 28 after implantation. TTE was defined as the time from the beginning of treatment to the day of euthanasia due to $\mathrm{MM}$ disease progression. Analysis was performed using Kaplan-Meier log rank survival analysis and the Holm-Sidak method of multiple comparisons. All animal experiments were performed according to the institutional guidelines and the protocol approved by the institutions.

\section{Immunohistochemistry and immunofluorescence}

Immunohistochemical studies were performed on selected tumors excised from treated and control mice. After fixation for 24 $\mathrm{h}$ in paraformaldehyde $10 \%$, a tissue microarray (TMA) was performed with a manual tissue arrayer (Beecher Intruments, Sun
Prairie, WI, USA), including two representative cylinders of each sample of $1 \mathrm{~mm}$ of diameter. TMA sections $3 \mu \mathrm{m}$ thick were obtained. After deparaffinization in xylene and rehydratation in increasing concentrations of ethanol, antigen retrieval, and incubation with the different primary and secondary antibodies was performed with a semi-automatic Dako Autostainer (DAKO, Carpinteria, CA, USA) system. Primary antibodies included anticleaved PARP, anti-cleaved caspase-3 (Cell Signaling, Boston, MA, USA), and anti-Ki67 (Thermo Scientific, Fremont, CA, USA). HRPconjugated secondary antibody was from Envision system, (DAKO ref K5007). Staining was performed with the IHC DAB MAP system (Ventana Medical Systems, Tucson, AR, USA). Sections were then counterstained with hematoxylin and analyzed by standard light microscopy.

For the immunofluorescence analysis, primary antibodies included anti-cleaved PARP, anti-pH2AX and Ac-Histone H4 (Cell Signaling, Boston, MA); for the in vivo BrdU analysis, $30 \mathrm{mg} / \mathrm{g}$ of BrdU (Sigma) mixed with 5-fluoro-2'- deoxyuridine (FdU, Sigma; $3 \mathrm{mg} / \mathrm{g}$ ) were intraperitoneally injected $1 \mathrm{~h}$ before the sacrifice of the mice. After the excision of the tissues, tumors were washed and DNA was denatured with $2 \mathrm{~N} \mathrm{HCl}$ and sections were incubated with biotinylated rat anti-BrdU antibody (Accurate Chemical and Scientific Corporation, NY, USA) or with rat anti-IgG Cy2 or Cy3-conjugated (Jackson ImmunoResearch Laboratories, Suffolk, UK) for the remaining antibodies. Samples were analyzed with a confocal laser microscope (Leica TCS SP2).

\section{Analysis of panobinostat on tumor-mediated bone damage}

Bone density was determined from three-dimensional reconstruction of images of the proximal tibia obtained using a high-resolution MicroCT scanner (VivaCT40, Scanco, Switzerland). Bone density was calculated as the percent bone volume divided by total volume (\%BV/TV). Trabecular \%BV/TV of the proximal tibia was determined within a $0.735 \mathrm{~mm}$ region.

Bone density was determined in all groups upon onset of clinical signs of MM in vehicle-treated animals. Animals in each group were imaged over 2 days.

\section{Results}

\section{Panobinostat potentiates the efficacy of standard of care anti-myeloma agents in both in vitro and ex vivo settings}

In previous studies we already showed that panobinostat synergized with conventional antimyeloma agents in double combinations. ${ }^{14}$ Now we have extended our work to investigate the optimal triple combinations of panobinostat to be assessed in mouse models in vivo and, hopefully, in the clinic.

As steroids have been the gold standard in the treatment of MM for many years, the double combination of panobinostat and dexamethasone was chosen as a backbone for all triple combinations. Subsequently the most active agents were combined with these two agents in triple combinations. Interestingly, when either of the two recently approved agents for relapsed/refractory MM (bortezomib and lenalidomide) were added to the combination of panobinostat + dexamethasone in MM1S cells, the efficacy was clearly superior to the respective individual agents or double combinations.

The synergy/additivity of these double and triple combinations was analyzed using the Chou-Talalay method. For this purpose, MM1S cells were treated with different doses of these drugs in monotherapy, and in double and triple 
combinations. Figures $1 \mathrm{~B}$ and $1 \mathrm{C}$ represent the dose effect curves and the algebraic estimate of the combination index (CI) of the double (1B) and triple (1C) combinations. As can be observed in the right panel of the figures (algebraic estimate of the CI), two double combinations, panobinostat + lenalidomide and lenalidomide + dexamethasone, displayed very synergistic results with CI less than 0.1 for all the doses tested. The remaining double combinations showed CI in the synergistic range, although, in the case of bortezomib combinations, these results were only observed when high doses of this compound were employed. Regarding triple combinations (Figure 1C), both of them were very synergistic in all the ranges of doses used: panobinostat + bortezomib + dexamethasone (PBD) showed a CI of 0.1-0.2, whereas panobinostat + lenalidomide + dexamethasone (PLD) displayed a better CI $(<0.1)$, probably because of the low activity of lenalidomide when used as a single agent.

The activity of both triple combinations was further confirmed ex vivo by using freshly isolated plasma cells from three $\mathrm{MM}$ patients including one patient with a highly resistant secondary plasma cell leukemia. As illustrated in Figure $2 \mathrm{~A}$, although the double combinations of panobinostat plus either lenalidomide or bortezomib were highly effective, the antitumor activity increased even further with triple combinations in all cases, especially in the patient with plasma cell leukemia.

In order to gain insights into the mechanism of the decrease in viability of the triple combinations observed in the MTT assays, we performed annexin V staining experiments by flow cytometry (Figure 2B), which indicate apoptotic induction. The antiproliferative activity was further analyzed by evaluating the effects on the cell cycle profile. Treatment with either PBD or PLD for $48 \mathrm{~h}$ induced an increase in the percentage of cells in G0/G1 with a decrease of those in proliferative phases ( $\mathrm{S}$ and G2/M phases) (Figure $2 \mathrm{C}$ ). In contrast, single-agent treatment did not induce any effect on the cell cycle profile, possibly due to the use of suboptimal doses.

\section{Unique genes are deregulated by triple combinations}

We hypothesized that the synergy observed in triple combinations was the result not only of the sum of the genes activated by the individual agents but also of the deregulation of additional genes. To test this hypothesis, we used oligonucleotide microarrays to analyze changes in the gene expression profile (GEP) of MM1S cells after treatment with panobinostat, dexamethasone, bortezomib, lenalidomide, and the two triple combinations under study (PBD and PLD). In order to obtain comparable results, the doses and times of treatment were fixed to achieve 20-25\% of apoptosis for each treatment. Accordingly, cells were treated with single agents for $48 \mathrm{~h}$, while in the triple $\mathrm{PBD}$ and PLD combinations, although the dose of the individual drugs was the same, the incubation times were reduced to 24 and $26 \mathrm{~h}$ in order to maintain the $20-25 \%$ rate of apoptosis. GEP analysis of treated MM1S cells identified a total of 466 genes significantly affected after treatment with panobinostat; 219 with dexamethasone; 156 with bortezomib and 695 with lenalidomide; while with the triple combinations of PBD and PLD, despite inducing the same percentage of apoptosis as the agents in monotherapy and despite using a shorter time of incubation, the number of deregulated genes was 1,206 and 2,008 respectively, figures significantly higher than the corresponding sum for the individual drugs. Thus, the comparative analyses of the GEP identified a set of 895 and 1323 genes exclusively deregulated in cells treated with PBD and PLD, respectively. In line with the results of the annexin $\mathrm{V}$ and cell cycle experiments, the most significantly deregulated functional categories corresponded to cell cycle and apoptosis (Online Supplementary Tables S1 and S2).

\section{Triple combinations of panobinostat have superior activity compared to single agents and double combinations in a murine model of multiple myeloma}

Given the strong anti-myeloma action of the triple combinations, we explored whether this in vitro effect was translated into in vivo model systems. As a preliminary step, we analyzed the in vivo antimyeloma activity of panobinostat as a single agent in two different murine xenograft models: one of a subcutaneous plasmacytoma, and another of a disseminated myeloma using luciferized MM1S cells. SCID mice bearing human subcutaneous plasmacytomas were randomized to receive PBS or panobinostat, and, as shown in Figure $3 \mathrm{~A}$, treatment significantly decreased the growth of human plasmacytomas $(P<0.05)$ without important toxicity. This inhibition of tumor growth correlated with an important improvement in time to endpoint (TTE): median TIE of 30 versus 70 days in animals treated with the vehicle and panobinostat, respectively $(P<0.001)$ (Figure 3B).

In the disseminated luciferized model, the efficacies of three different doses of panobinostat were assessed: 5, 10 and $20 \mathrm{mg} / \mathrm{kg}$ i.p. 5 days weekly. In all three dosed groups, panobinostat demonstrated a clear benefit of decreased tumor burden, as measured by photons/sec of bioluminescence and statistically significant differences were seen for groups treated with 10 and $20 \mathrm{mg} / \mathrm{kg}$ doses, as compared with the vehicle control group (Figure 3C). Panobinostat treatment also significantly improved TTE, especially at the $20 \mathrm{mg} / \mathrm{kg}$ dose. As shown in Figure 3D, we observed a median TTE of $37,44,50$, and 68 days for the vehicle, 5, 10 and $20 \mathrm{mg} / \mathrm{kg}$ panobinostat groups, respectively. All treatment groups displayed statistically significant differences in terms of survival $(P<0.05)$ as compared to the vehiclereceiving group.

Furthermore, the efficacy of panobinostat in well-established tumors was assessed in an in vivo model of big plasmacytomas. Mice bearing large subcutaneous human plasmacytomas were treated with panobinostat at a dose of 10 $\mathrm{mg} / \mathrm{kg}$ i.p. for 5 days weekly for 2 weeks. A marked decrease in tumor volume was observed in all animals following treatment (Figure 3E), with near complete regression in two of them (data not shown).

Tumors were excised for immunofluorescence analysis after 2 weeks of treatment. A representative example of analysis of the molecular effects in situ is shown in Figure 3F. An increase on histone $\mathrm{H} 4$ acetylation, decreased proliferation, as assessed by BrdU uptake, and increased apoptosis, as assessed by cleavage of caspase-3, were observed. Altogether, these results support the antiproliferative and proapoptotic effects of panobinostat in vivo.

Next, we assessed the in vivo activity of the two triple combinations in the subcutaneous human plasmacytoma model. Animals were randomized to receive vehicle (PBS), the triple combinations of panobinostat + bortezomib + dexamethasone (PBD) and panobinostat + lenalidomide + dexamethasone (PLD), or the agents in monotherapy and in double combinations (Figure 4). Suboptimal doses of 
bortezomib (0.1 mg/kg i.p., 5 days weekly), lenalidomide $(15 \mathrm{mg} / \mathrm{kg}$ i.p., 5 days weekly) and dexamethasone (1 $\mathrm{mg} / \mathrm{kg}$ ip, 5 days weekly) were used to assess the potential synergistic effects of the combinations. The dose of panobinostat was initially established at $10 \mathrm{mg} / \mathrm{kg}$ i.p., 5 days weekly and was decreased to $5 \mathrm{mg} / \mathrm{kg}$ after 21 days of treatment, due to the high level of anti-tumor activity observed. Neither bortezomib nor lenalidomide was able to potentiate the effect of dexamethasone (Figure 4). This lack of additive effect may be due to the low doses of bortezomib and lenalidomide used. In contrast, panobinostat significantly potentiated the effects of dexamethasone (Figure 4 A-D), bortezomib (Figure 4 A-B), and lenalidomide (Figure 4 C-D). Moreover, both triple combinations (PBD and PLD) demonstrated significantly greater effects than any of the double combinations, with respect to tumor growth inhibition (Figure $4 \mathrm{~A}, \mathrm{C}$ ). The median \pm SE of tumor volumes for the panobinostat, PBD and PLD groups at day 60 of treatment were $2118 \pm 229 \mathrm{~mm}^{3}, 524 \pm 114 \mathrm{~mm}^{3}$ and $526 \pm 54 \mathrm{~mm}^{3}$ respectively $(P<0.05)$. This benefit in the decrease of tumor burden for the animals treated with triple combinations correlated with a significant increase in survival, as shown in the Kaplan-Meier curves demonstrating median survival of 70, 88 and 88 days for the panobino-

A
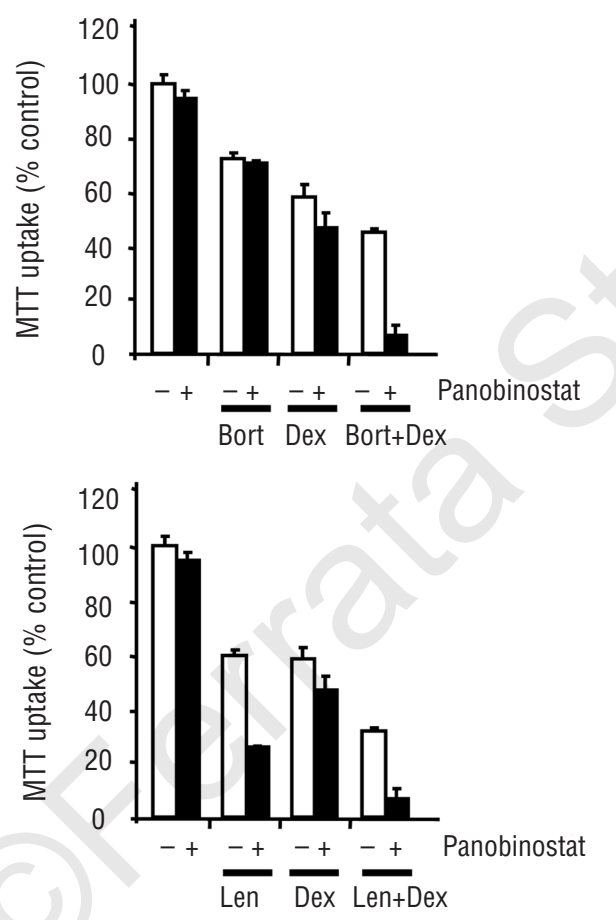

Figure 1. Efficacy of the in vitro combinations of panobinostat with other anti-myeloma agents in MM. (A) MTT studies of the double and triple combinations of panobinostat $(1 \mathrm{nM})$ with dexamethasone (5 $\mathrm{nM})$ and lenalidomide $(0.5 \mu \mathrm{M})$ or bortezomib $(2 \mathrm{nM})$ in the MM1S cell line after $72 \mathrm{~h}$ of treatment. Bars represent standard deviation of experiments repeated in quadruplicate. (B) and (C) Quantification of the potency of the different double $(B)$ and triple $(C)$ combinations using the Chou-Talalay method as described in the Design and Methods section. The left panel shows the dose-effect curve of the double combinations as compared to the dose-effect of the agents in monotherapy (B) and of the triple compared with the doubles combinations $(C)$. In the right panel, the $(x)$ indicates the observed $\mathrm{Cl}$ of each combination in the experiments performed and the line gives an estimation of the $\mathrm{Cl}$ for these combinations.
B

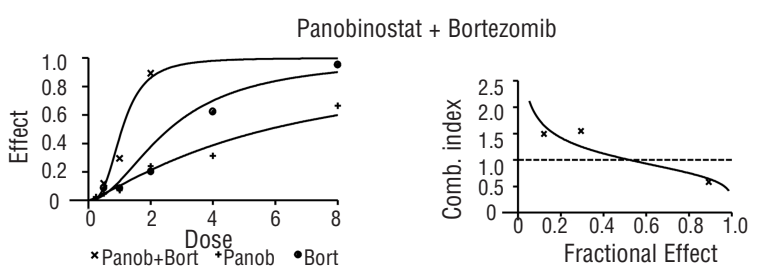

Panobinostat + Lenalidomide
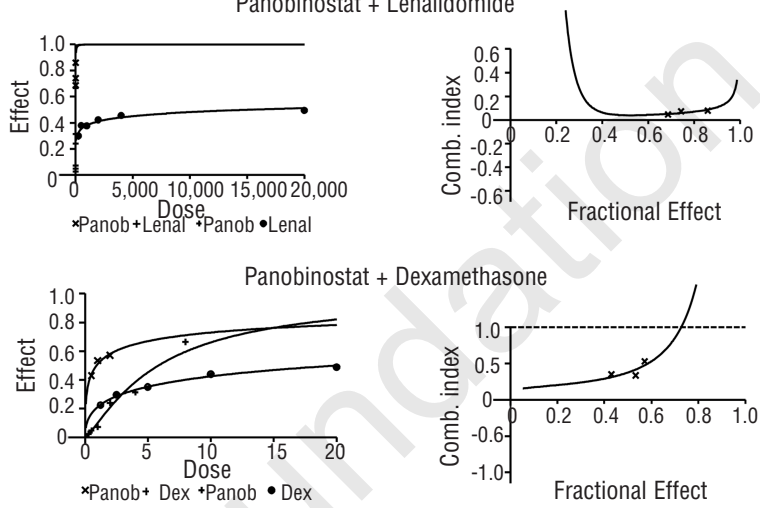

Bortezomib + Dexamethasone
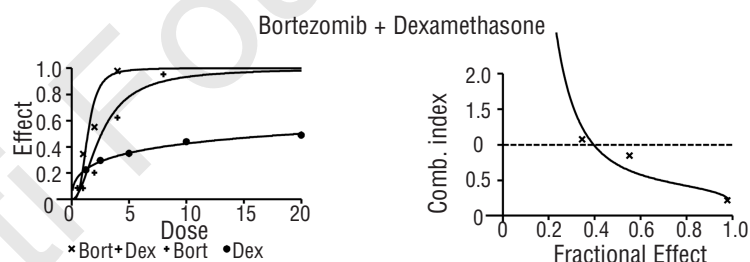

Lenalidomide + Dexamethasone

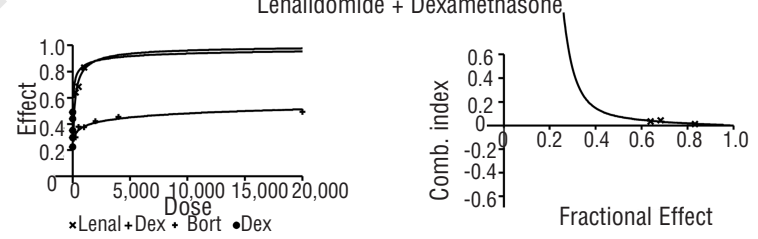

C Panobinostat + Bortezomib + Dexamethasome

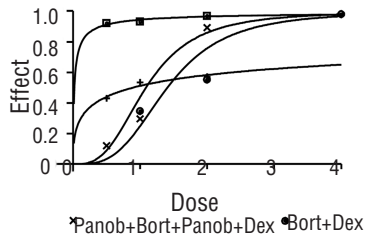

Panob+Bort+Panob+

四anob+Bort+Dex

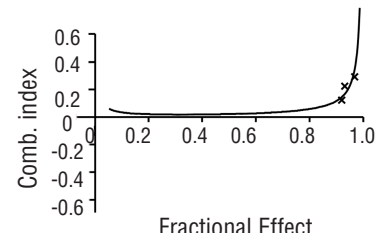

Fractional Effect

Panobinostat + Lenalidomide + Dexamethasome
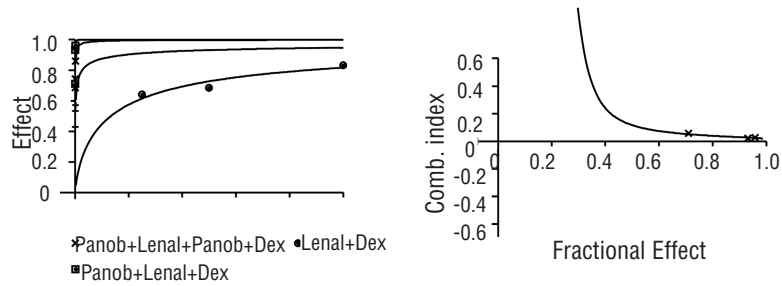

Fractional Effect 
stat, PBD and PLD groups, respectively (log rank, $P<0.001$ ) (Figure 4B, D). Moreover, when the TTE of mice treated with the triple combinations was compared with the TTE of mice receiving the respective double combinations, there was also a statistically significant advantage for the triple combinations in all cases ( $P$ values ranging between 0.01 and 0.04 ) (Figure $4 \mathrm{~B}, \mathrm{D})$. The triple combinations including bortezomib or lenalidomide appeared to be equally active, with no significant differences between them. Notably, only a slight decrease in body weight $(<10 \%$ as compared to the vehicle) was observed in the animals treated with the triple combinations (data not shown). Tumors were extracted for immunohistochemical analysis of proliferation markers and apoptosis (Figure 4E). Tumors from mice treated with triple combinations showed a decrease in expression of the cell proliferation antigen Ki-67, as well as increased expression of the apoptotic markers cleaved caspase-3 and PARP. Both combinations also increased the phosporylation of $\mathrm{H} 2 \mathrm{AX}$ indicating the induction of DNA double-strand breaks (DSB) with the combination. Together, these results support the antiproliferative and pro-apoptotic effects of the triple panobinostat combinations as well as a role of the combination in inducing DNA damage.

\section{Panobinostat reduces bone density loss in a disseminated multiple myeloma mouse model}

As bone disease is highly relevant into both the pathogenesis of $\mathrm{MM}$ and the quality of life of $\mathrm{MM}$ patients, the effect of panobinostat on bone disease was explored. As shown in Figure 5, panobinostat treatment significantly reduced bone density loss in a systemic MM xenograft mouse model. Animals with systemic disease treated with panobinostat displayed reduced trabecular (Figure 5A) bone damage as compared with vehicle-treated animals in a dose-related fashion, as assessed by Micro CT. The mean trabecular \%BV/TV for vehicle-treated animals was $3.2 \pm 0.6 \%$ (mean \pm SEM), while in animals treated with panobinostat at 5,10 , or $20 \mathrm{mg} / \mathrm{kg}$ it was $6.7 \pm 0.9 \%$, $8.6 \pm 0.8 \%$, and $12.3 \pm 1 \%$, respectively (Figure $5 \mathrm{~B}$ ), with statistically significant differences $(P<0.05)$ between the control, 10 , and $20 \mathrm{mg} / \mathrm{kg}$ doses.

\section{Discussion}

Combinations of anti-cancer compounds have traditionally been based on empiric decisions without a clear experimental background. This practice has resulted, in some cases, in very effective associations that have become stan-

A
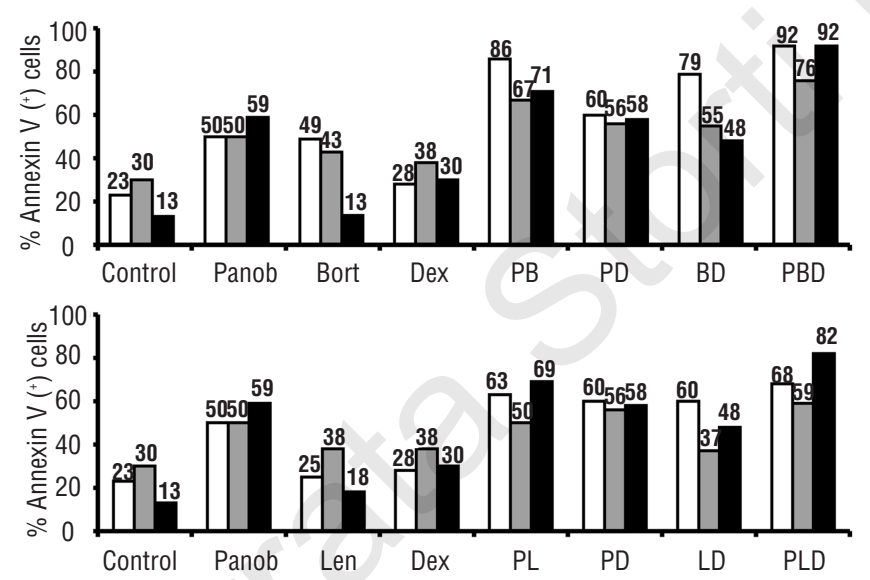

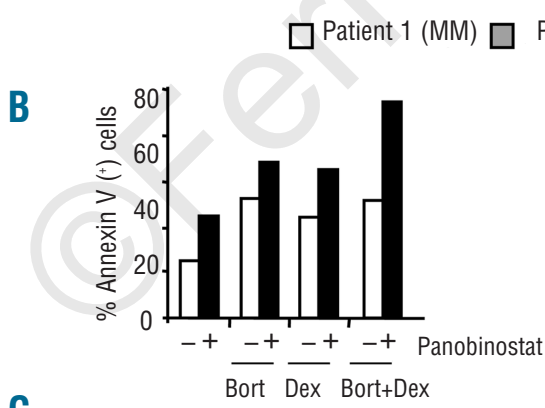

Patient 2 (MM

Patient 3 (PCL)

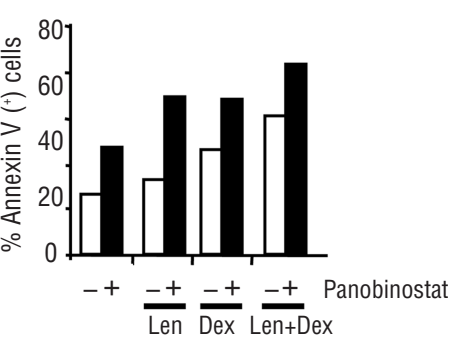

C

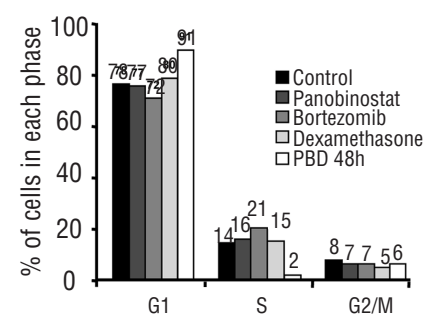

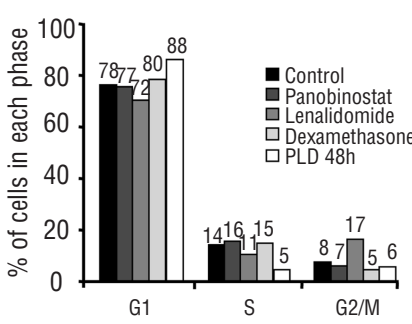

Figure 2. Ex vivo antimyeloma efficacy of the triple combinations (PBD and PLD) and apoptotic and antiproliferative activity. (A) Bone marrow samples from two patients with MM and a patient with plasma cell leukemia (PCL) were treated ex vivo for $24 \mathrm{~h}$ with panobinostat $(20$ $\mathrm{nM})$, dexamethasone $(40 \mathrm{nM})$, bortezomib (5 nM), and lenalidomide (10 $\mu \mathrm{M})$ in monotherapy and in double and triple combinations. Samples were incubated with annexin $\mathrm{V}$ and CD38, CD45, CD56 monoclonal antibodies to analyze the induction of apoptosis in the clonal population of plasma cells (B) Annexin V studies of the double and triple combinations of panobinostat (1 nM) with dexamethasone $(10 \mathrm{nM})$ and bortezomib (2 $\mathrm{nM})$ or lenalidomide $(0.5 \mu \mathrm{M})$ in the MM1S cell line after $48 \mathrm{~h}$ of treatment. (C) MM1S cells were incubated with panobinostat $(7 \mathrm{nM})$, dexamethasone $(0.9 \mu \mathrm{M})$, lenalidomide $(1 \mu \mathrm{M})$ and bortezomib (3 $\mathrm{nM}$ ) as single agents for $48 \mathrm{~h}$ and in triple combinations for 24 and 48 $\mathrm{h}$ and the cell cycle profile was examined by flow cytometry after propidium lodide staining. Bars indicate the percentage of cells in each phase of the cell cycle from a representative example. 
dard of care for some malignancies, but in other cases, these combinations have not shown the expected benefits in terms of anticancer activity or have resulted in unacceptable side effects. More recently, the lack of activity observed with many of the novel targeted single agents directed against specific mechanisms of tumor cell growth and survival has encouraged the investigation of scientifically-based combinations. In this regard, in vitro and in vivo studies are essential in the search for optimal drug combinations with a double aim: to exhibit significantly better efficacy than that observed with the agents in monotherapy, and to display an adequate toxicity profile. Moreover, since the combination of proteasome inhibitors or immunomodulatory drugs (IMiD) plus corticosteroids has become the backbone of MM treatment, we propose that the efficacy of new experimental agents should be investigated in this context in order to explore whether triple combinations are able to improve the efficacy of these double combinations of agents.

On this background we report on the anti-MM activity of the combination of panobinostat, a novel deacetylase inhibitor, with dexamethasone and either one of two recently approved drugs with well established anti-MM action: the proteasome inhibitor bortezomib ${ }^{20,21}$ and the immunomodulatory drug lenalidomide. ${ }^{22,23}$ As a first step, we showed that both combinations (PBD and PLD) had a consistent significant advantage over the respective agents used either in monotherapy or in double combinations in MM cell lines, and in freshly isolated plasma cells from MM patients. The quantification of the potency of all these combinations (both double and triple combinations) by the Chou-Talalay method showed clear in vitro synergy for all of them, especially those including lenalidomide, probably due to the pattern of activity of this drug as a single agent. These results were confirmed in vivo in murine models, in which a statistically significant survival advantage was observed for those mice treated with the triple combinations as compared with any of the double combinations. Noteworthy, no significant difference was observed between the combination that included bortezomib and the one that included lenalidomide in terms of decrease in tumor growth and in terms of time to clinical endpoint.

This marked activity of both triple combinations correlated with a completely unique profile of deregulated genes upon treatment, as compared to the sum of the genes deregulated by the three agents as monotherapy. In this regard, triple combinations resulted in specific deregulation of approximately 1,000 genes (895 and 1,323 for PBD and PLD, respectively) that were not affected after treatment with any of the agents in monotherapy.
A

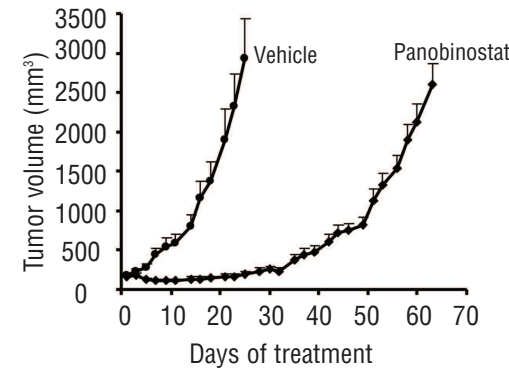

C

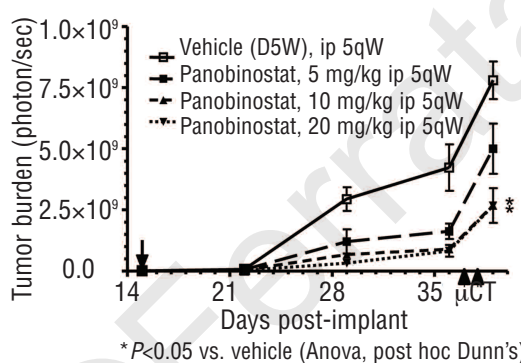

${ }^{*} P<0.05$ vs. vehicle (Anova, post hoc Dunn's)

E

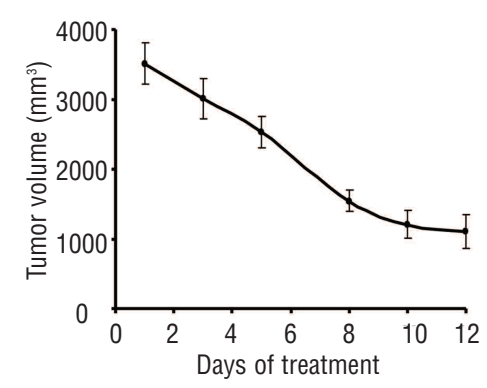

B

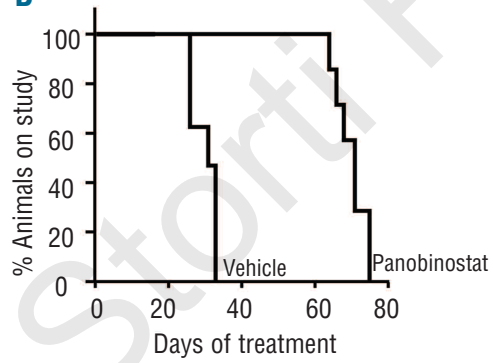

D

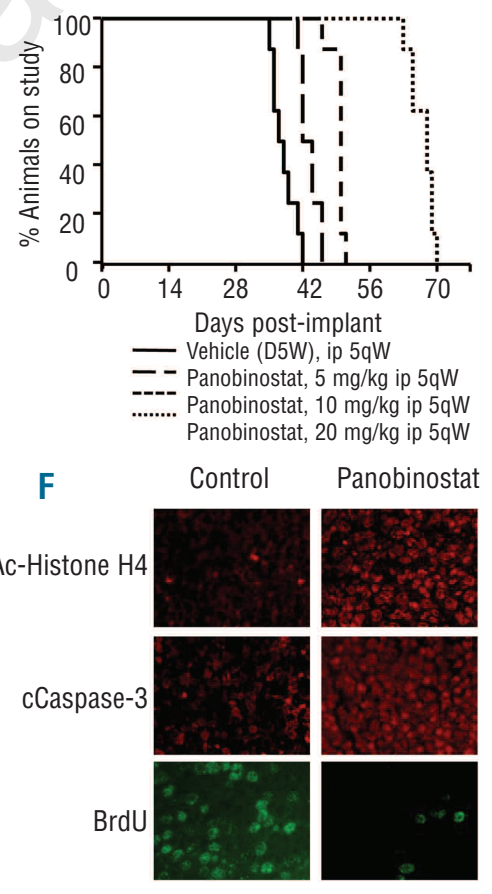

Figure 3. Efficacy of single-agent panobinostat in xenograft models of MM. $(A, B)$ Panobinostat activity in a human subcutaneous plasmacytoma model in NOD-SCID mice. Mice were randomized to receive vehicle control or panobinostat at a dose of 10 $\mathrm{mg} / \mathrm{kg}$ i.p. 5 days weekly for 21 days or 5 $\mathrm{mg} / \mathrm{kg}$ on the same schedule on subsequent days. Panobinostat treatment resulted in inhibition of tumor growth $(A)$ and increased survival (B) as compared with the control group $(P<0.05)$. Median survival was 70 days versus 30 days for the panobinostat treated and vehicle groups, respectively $(P<0.05)$. (C,D) Efficacy of panobinostat in a murine model of disseminated human myeloma. Treatment was initiated 15 days after implantation with the doses indicated in the figure. (C) Panobinostat treatment resulted in dose-related reduction in tumor burden. The tumor burden (\%T/C) on day 39 , post-implant, following treatment with panobinostat at 5,10 , or $20 \mathrm{mg} / \mathrm{kg}$ was 64 , 34 , and $34 \%$ of that of animals treated with vehicle. (D) Panobinostat treatment increased the median time to clinical endpoint (Kaplan-Meier). The median TTE for vehicle treated animals was 37 days. The median TTE for panobinostat dosed at 5, 10 and $15 \mathrm{mg} / \mathrm{kg}$ was 43,50 and 68 days, respectively $(P<0.05$ for all pair-wise comparisons, Holm-Sidak). (E, F) Mice with large plasmacytomas were treated with panobinostat at a dose of $10 \mathrm{mg} / \mathrm{kg}$. Tumors which had decreased in volume by at least $50 \%$ following 10 days of treatment were collected and analyzed by immunofluorescence for the presence of acetylated histone $\mathrm{H} 4$, cleaved caspase-3 and BrdU uptake (Pictures 40x). Panobinostat treatment increased expression of acetylated histone $\mathrm{H} 4$ and cleaved caspase-3, and decreased BrdU nuclear immunofluorescence. (Magnification 40x) 
A

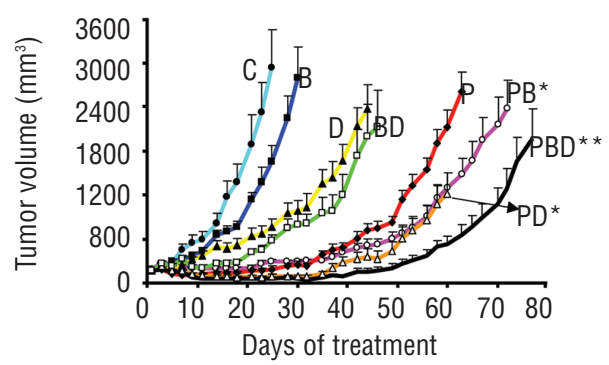

B

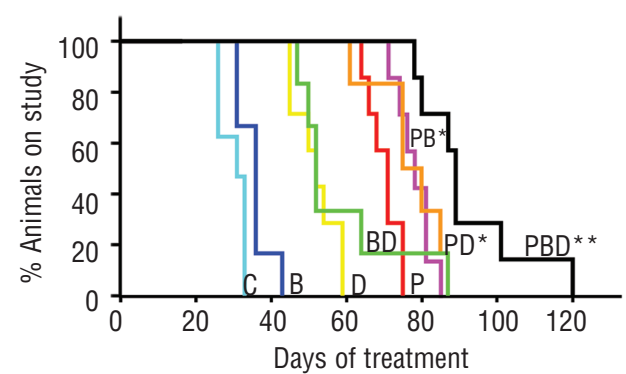

C

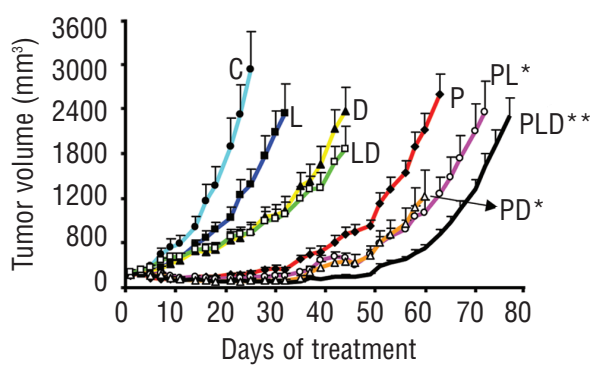

D

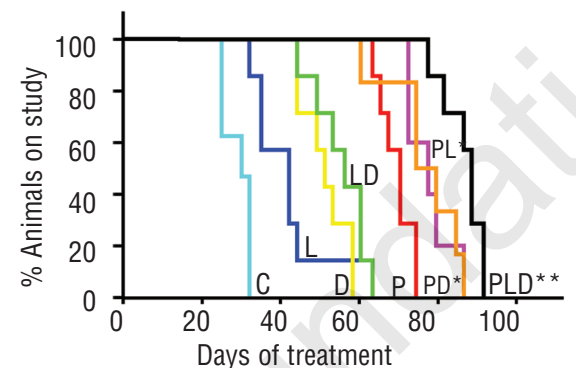

E

Control Panobinostat Bortezomib Dexamethasone PBD

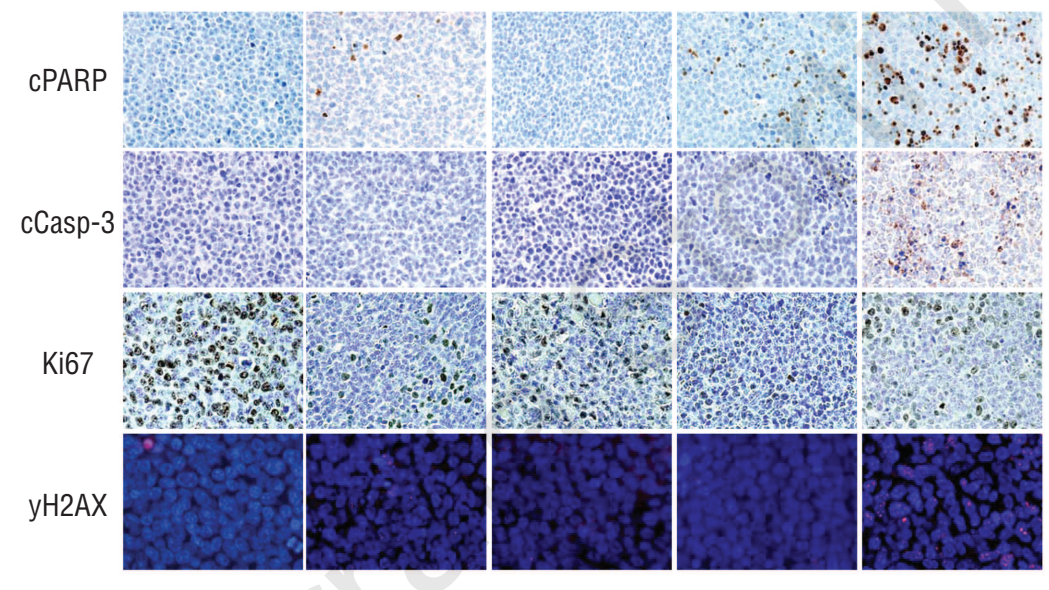

Control Panobinostat Lenalidomide Dexamethasone PLD

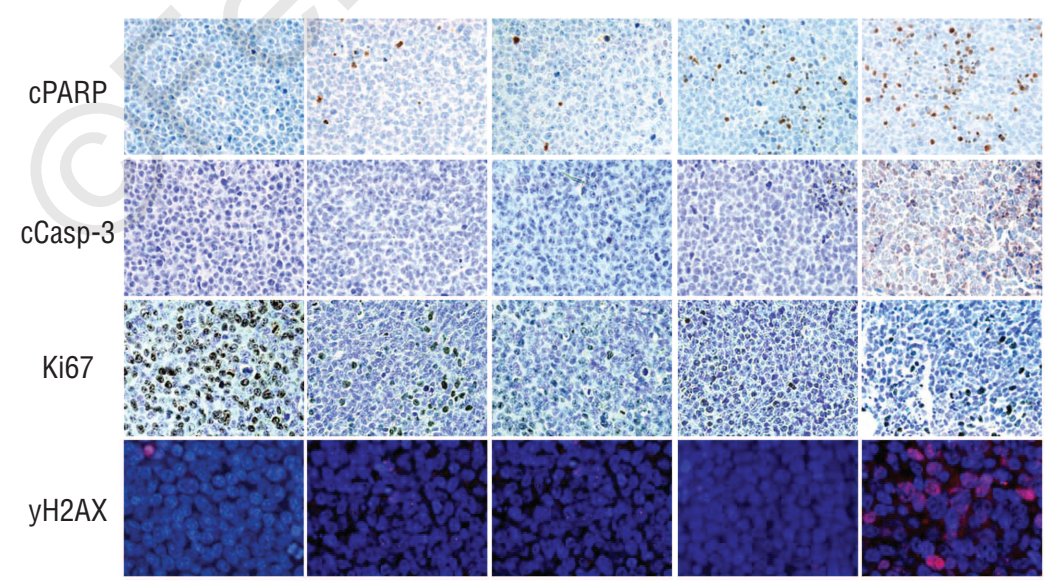

Figure 4. In vivo efficacy of triple combinations of panobinostat with dexamethasone and either bortezomib (PBD) or lenalidomide (PLD). Tumor-bearing mice were treated with vehicle control, panobinostat $(10 \mathrm{mg} / \mathrm{kg}$ i.p. for the first 21 days and $5 \mathrm{mg} / \mathrm{kg}$ the subsequent days), bortezomib $(0.1 \mathrm{mg} / \mathrm{kg}$ i.p. 5 days weekly), lenalidomide $(15 \mathrm{mg} / \mathrm{kg}$ i.p., 5 days weekly) and dexamethasone $(1 \mathrm{mg} / \mathrm{kg}$ ip, 5 days weekly) in monotherapy or in double and triple combinations. (A,C) Tumor volumes of MM1S plasmacytomas following treatment with single agents, double and triple combinations with bortezomib (A) and lenalidomide (C). Statistical differences between groups were analyzed with one-way analysis of variance, and statistical significance was defined as $P<0.05$. Bars indicate standard error. $(B, D)$ Survival was analyzed in a Kaplan Meier curve, and statistical difference was defined as $P<0.05$ in the log rank test. (*) indicates statistically significant differences between double combinations and their respective agents in monotherapy. $(* *)$ indicates statistically significant differences between triple combinations and their respective double combinations. (E) Immunohistochemical analyses with anti-cleaved caspase-3, anti-cleaved-PARP, anti-Ki-67 and immunofluorescence studies with phospho-H2AX were performed in selected tumors obtained from mice receiving the vehicle control and mice receiving triple combinations. Representative images demonstrate the differences in expression of these particular markers induced by the in vivo treatment with triple combinations. (Magnification 40x). 
Several genes involved in pathways such as apoptosis or cell cycle were found among the genes distinctively deregulated by the triple combinations, and these results were corroborated in functional experiments assessing cell proliferation and apoptosis. Both triple combinations (PBD and PLD) potently induced an increase in annexin V staining and a blockade of cell cycle, and these effects, were, once more, more obvious in cells treated with the triple combinations than with any of the respective double combinations. Immunohistochemical studies in tumors excised from mice treated with both triple combinations validated the results, showing an increase in apoptotic proteins and a decrease in proliferative markers in tumors excised from mice treated with both triple combinations. Moreover, a role for DNA damage in the mechanism of both triple combinations is suggested by the increase of pH2AX observed after treatment.

A second aspect of the preclinical evaluation of novel combinations is the assessment of the toxicity profile. Both PBD and PLD, besides showing very potent antimyeloma activity in the animal studies, displayed very low toxicity with only a very modest loss of body weight in the treated animals (less than 10\%), suggesting that these drug combinations can reach therapeutic serum concentrations without inducing significant adverse effects.

Patients with multiple myeloma may present with two different patterns of disease; the most frequent one corresponds to systemic disease with bone marrow infiltration and general symptoms; the second is characterized by the

A

Trabecular bone

vehicle

panobinostat

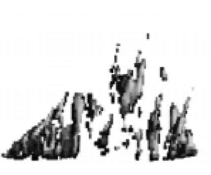

B

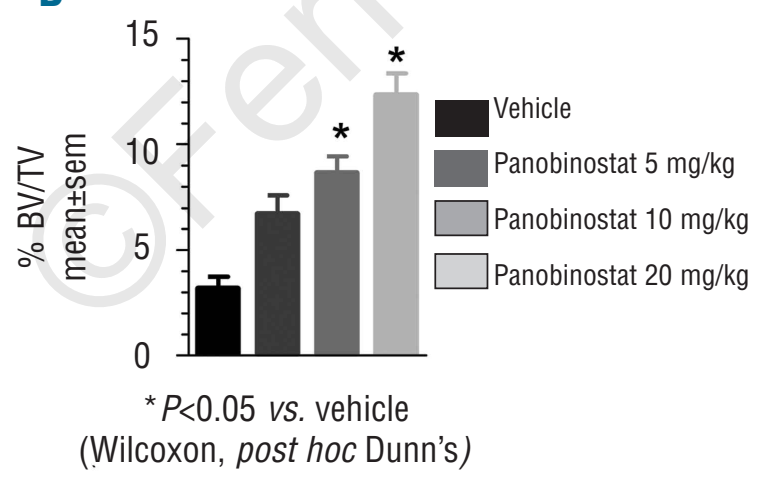

Figure 5. Panobinostat reduces bone density loss in a disseminated MM xenograft mouse model (A) Representative 3-D MicroCT image from trabecular bone in MM xenograft mice treated with vehicle or panobinostat at $15 \mathrm{mg} / \mathrm{kg}$ i.p. 5 times weekly. (B) The trabecular bone density of MM xenograft mice was determined by MicroCT on days 37-38, post-implant, following treatment, as indicated. presence of localized plasmacytomas that may or may not be associated with disseminated disease. It is important to note that, following the introduction of novel agents and the use of allogeneic bone marrow transplantation, we observe not only a prolongation of survival, but also an increase in the frequency of extramedullary disease..$^{24,25}$

Panobinostat demonstrated significant efficacy in the two different animal models of $\mathrm{MM}$; the intravenous model that represents disseminated disease, and a subcutaneous plasmacytoma model, which partially resembles the extramedullary disease. The activity of panobinostat in both MM models suggests that the therapeutic effects can be achieved systemically, not only in the bone marrow, but also in subcutaneous tissues.

Myeloma bone disease is one of the most debilitating complications of $\mathrm{MM}$, and the increase in life expectancy observed in the last years for $\mathrm{MM}$ patients ${ }^{26,27}$ has made preserved quality of life a goal for MM therapy. Moreover, recent reports suggest that the myeloma bone disease may play an important role in $\mathrm{MM}$ pathogenesis. ${ }^{28,29}$ This manifestation is the result of an increased destruction ${ }^{30,31}$ of bone that cannot be compensated by new bone formation. ${ }^{31-33}$ Therefore, the efficacy of novel agents in managing myeloma-related bone disease has become of particular interest. As reviewed by Terpos et al., ${ }^{34}$ several studies have demonstrated the effect of IMID (thalidomide and lenalidomide) in reducing osteoclast activity while the proteasome inhibitor, bortezomib, appears to have a dual effect, inducing a simultaneous reduction in bone resorption and an increase of bone formation markers. ${ }^{34,35}$ In our in vivo model of disseminated disease, we demonstrated the efficacy of panobinostat in controlling myeloma-related bone disease with a clear dose-dependent decrease in trabecular bone loss.

In summary, our results indicate that panobinostat demonstrates promising activity in $\mathrm{MM}$ via the reduction of tumor burden, inhibition of disease progression, and preservation of bone integrity. Triple combinations of panobinostat + dexamethasone and either bortezomib or lenalidomide are safe and display promising anti-myeloma efficacy which is based on the activation of a different group of genes than those triggered by the sum of the agents in monotherapy. These studies have provided the rationale for the activation of two clinical trials with these triple combinations (panobinostat + bortezomib \pm dexamethasone and panobinostat + lenalidomide + dexamethasone) in patients with relapsed/refractory MM.

\section{Authorship and Disclosures}

EMO and DV designed and performed research, analyzed data, and wrote the paper; PM and EA designed and performed research, analyzed data, and contributed to the write-up of the paper; EC, DF-L, MG, LS-S and TH-I performed research and analyzed data; PA, WS and YY contributed research tools, analyzed data and contributed to the write-up of the paper; AP and JFS-M designed research, analyzed data, and contributed to the write-up of the paper. PA, WS and YY are employees of Novartis Pharmaceuticals. EMO and JFSM have participated in advisory boards related to the topic of the manuscript and received honoraria from Novartis Pharmaceuticals. EMO was supported by the 'Plan Nacional de Investigación Científica, Desarrollo e Innovación Tecnológica (I+D+I)' and by 'Instituto de Salud Carlos III-Fondo de Investigación Sanitaria' with expedient 
number 400001. EC was supported by a grant from the Carolina Foundation (BBVA) and is a postgraduate fellow in health sciences in the "Facultad de Ciencias Medicas de la Santa Casa de Misericórdia de São Paulo". MG was supported by the "Plan Nacional de Investigación Científica,
Desarrollo e Innovación Tecnológica $(\mathrm{I}+\mathrm{D}+\mathrm{I})^{\prime}$ and by 'Instituto de Salud Carlos III-Fondo de Investigación Sanitaria' with expedient number 05/0279. PM was supported by the FIS-FEDER through projects to JSM, and a Spanish Myeloma Network Program (G03/136).

\section{References}

1. Ocio EM, Mateos MV, Maiso P, Pandiella A, San-Miguel JF. New drugs in multiple myeloma: mechanisms of action and phase I/II clinical findings. Lancet Oncol. 2008; 9(12):1157-65.

2. Hanahan D, Weinberg RA. The hallmarks of cancer. Cell. 2000;100(1):57-70.

3. Hallek M, Eichhorst BF. Chemotherapy combination treatment regimens with fludarabine in chronic lymphocytic leukemia. Hematol J. 2004;5 (Suppl 1):S20-S30.

4. Kyrgiou M, Salanti G, Pavlidis N, Paraskevaidis E, Ioannidis JP. Survival benefits with diverse chemotherapy regimens for ovarian cancer: meta-analysis of multiple treatments. J Natl Cancer Inst. 2006; 98(22):1655-63.

5. Mauri D, Polyzos NP, Salanti G, Pavlidis N, Ioannidis JP. Multiple-treatments metaanalysis of chemotherapy and targeted therapies in advanced breast cancer. J Natl Cancer Inst. 2008;100(24):1780-91.

6. Srikanth M, Davies FE, Morgan GJ. An update on drug combinations for treatment of myeloma. Expert Opin Investig Drugs. 2008:17(1):1-12

7. Mahlknecht U, Hoelzer D. Histone acetylation modifiers in the pathogenesis of malignant disease. Mol Med. 2000;6(8):623-44

8. Timmermann S, Lehrmann H, Polesskaya A, Harel-Bellan A. Histone acetylation and disease. Cell Mol Life Sci. 2001;58(5-6):72836.

9. Mitsiades CS, Hayden PJ, Anderson KC, Richardson PG. From the bench to the bedside: emerging new treatments in multiple myeloma. Best Pract Res Clin Haematol. 2007;20(4):797-816.

10. Pan LN, Lu J, Huang B. HDAC inhibitors: a potential new category of anti-tumor agents. Cell Mol Immunol. 2007;4(5):33743.

11. Ellis L, Pan Y, Smyth GK, George DJ, McCormack C, Williams-Truax $\mathrm{R}$, et al. Histone deacetylase inhibitor panobinostat induces clinical responses with associated alterations in gene expression profiles in cutaneous T-cell lymphoma. Clin Cancer Res. 2008;14(14):4500-10.

12. Ottmann OG, Spencer A, Prince HM, Bhalla KN, Fischer T, Liu A, et al. Phase IA/II study of oral panobinostat (LBH589), a novel pan- deacetylase inhibitor (DACi) demonstrating efficacy in patients with advanced hematologic malignancies. Blood. 2008;112(11):958a.

13. Catley L, Weisberg E, Kiziltepe T, Tai YT, Hideshima T, Neri P, et al. Aggresome induction by proteasome inhibitor bortezomib and alpha-tubulin hyperacetylation by tubulin deacetylase (TDAC) inhibitor
LBH589 are synergistic in myeloma cells. Blood. 2006;108(10): 3441-9.

14. Maiso P, Carvajal-Vergara X, Ocio EM, Lopez-Perez R, Mateo G, Gutierrez N, et al. The histone deacetylase inhibitor LBH589 is a potent antimyeloma agent that overcomes drug resistance. Cancer Res. 2006; 66(11):5781-9.

15. Galli M, Salmoiraghi S, Golay J, Gozzini A Bosi A, Crippa C, et al. A phase II multiple dose clinical trial of histone deacetylase inhibitor ITF2357 in patients with relapsed or progressive multiple myeloma: preliminary results. Blood. 2007;110(11):356aAbstract 1175

16. Richardson PG, Mitsiades CS, Colson $\mathrm{K}$ Reilly E, McBride L, Chiao J, et al. Final results of a phase I trial of oral vorinostat (suberoylanilide hydroxamic acid, SAHA) in patients with advanced multiple myeloma. Blood. 2007;110(11):357a-Abstract 1179

17. Wolf JL, Siegel D, Matous J, Lonial S, Goldschmidt H, Schmitt S, et al. A phase II study of oral panobinostat (LBH589) in adult patients with advanced refractory multiple myeloma. ASH Annual Meeting Abstracts. 2008;112(11):2774.

18. Carvajal-Vergara X, Tabera S, Montero JC, Esparis-Ogando A, Lopez-Perez R, Mateo $\mathrm{G}$, et al. Multifunctional role of Erk5 in multiple myeloma. Blood. 2005;105(11) 4492-9

19. Chou TC, Talalay P. Quantitative analysis of dose-effect relationships: the combined effects of multiple drugs or enzyme inhibitors. Adv Enzyme Regul. 1984;22:27-

20. Richardson PG, Sonneveld P, Schuster MW Irwin D, Stadtmauer EA, Facon T, et al Bortezomib or high-dose dexamethasone for relapsed multiple myeloma. $N$ Engl J Med. 2005;352(24):2487-98.

21. San Miguel JF, Schlag R, Khuageva NK, Dimopoulos MA, Shpilberg O, Kropff M et al. Bortezomib plus melphalan and prednisone for initial treatment of multiple myeloma. N Engl J Med. 2008;359(9):90617 .

22. Dimopoulos M, Spencer A, Attal M, Prince HM, Harousseau JL, Dmoszynska A, et al Lenalidomide plus dexamethasone for relapsed or refractory multiple myeloma. $\mathrm{N}$ Engl J Med. 2007;357(21):2123-32.

23. Weber DM, Chen C, Niesvizky R, Wang $M$, Belch A, Stadtmauer EA, et al. Lenalidomide plus dexamethasone for relapsed multiple myeloma in North America. N Engl J Med. 2007;357(21):2133 42

24. Minnema MC, van de Donk NW, Zweegman S, Hegenbart U, Schonland S, Raymakers R, et al. Extramedullary relapses after allogeneic non-myeloablative stem cell transplantation in multiple myeloma patients do not negatively affect treatment outcome. Bone Marrow Transplant. 2008;41(9):779-84.

25. Perez-Simon JA, Sureda A, Fernandez Aviles F, Sampol A, Cabrera JR, Caballero $\mathrm{D}$, et al. Reduced-intensity conditioning allogeneic transplantation is associated with a high incidence of extramedullary relapses in multiple myeloma patients. Leukemia. 2006;20(3):542-5.

26. Brenner H, Gondos A, Pulte D. Recent major improvement in long-term survival of younger patients with multiple myeloma. Blood. 2008;111(5):2521-6.

27. Kumar SK, Rajkumar SV, Dispenzieri A Lacy MQ, Hayman SR, Buadi FK, et al Improved survival in multiple myeloma and the impact of novel therapies. Blood. 2008;111(5): 2516-20

28. Yaccoby S, Wezeman MJ, Zangari $M$ Walker R, Cottler-Fox M, Gaddy D, et al. Inhibitory effects of osteoblasts and increased bone formation on myeloma in novel culture systems and a myelomatous mouse model. Haematologica. 2006;91(2): 192-9

29. Abe M, Hiura K, Wilde J, Shioyasono A, Moriyama K, Hashimoto $\mathrm{T}$, et al. Osteoclasts enhance myeloma cell growth and survival via cell-cell contact: a vicious cycle between bone destruction and myeloma expansion. Blood. 2004;104(8) 2484-91.

30. Pearse RN, Sordillo EM, Yaccoby S, Wong BR, Liau DF, Colman N, et al. Multiple myeloma disrupts the TRANCE/ osteoprotegerin cytokine axis to trigger bone destruction and promote tumor progression. Proc Natl Acad Sci USA. 2001;98(20): 11581-6.

31. Terpos E, Sezer O, Croucher P, Dimopoulos MA. Myeloma bone disease and proteasome inhibition therapies. Blood. 2007; 110(4):1098-104

32. Giuliani N, Rizzoli V, Roodman GD. Multiple myeloma bone disease: Pathophysiology of osteoblast inhibition. Blood. 2006;108(13):3992-6.

33. Taube T, Beneton MN, McCloskey EV Rogers S, Greaves M, Kanis JA. Abnormal bone remodelling in patients with myelomatosis and normal biochemical indices of bone resorption. Eur J Haematol. 1992;49 (4):192-8.

34. Terpos E, Dimopoulos MA, Sezer O. The effect of novel anti-myeloma agents on bone metabolism of patients with multiple myeloma. Leukemia. 2007;21(9):1875-84

35. Terpos E. Bortezomib directly inhibits osteoclast function in multiple myeloma: implications into the management of myeloma bone disease. Leuk Res. 2008, 32(11):1646-7. 